\title{
CFD SIMULATION ON WIND TURBINE BLADES WITH LEADING EDGE EROSION ${ }^{1}$
}

\author{
YAN WANG \\ School of Energy and Power Engineering, Lanzhou University of Technology, Lanzhou, China, and \\ Key Laboratory of Western Disaster and Environmental Mechanics of Ministry of Education, \\ Lanzhou University of Technology, Lanzhou, China \\ e-mail: yw2011@lzu.edu.cn \\ Liang Wang, Chenglin Duan, Jian Zheng, Zhe Liu, Gaosheng Ma \\ School of Energy and Power Engineering, Lanzhou University of Technology, Lanzhou, China
}

\begin{abstract}
Deep understanding on the impacts of leading edge erosion on the performance and flow characteristics of wind turbines is significant for the blade design and wind farms management. Pitting erosion and three levels of delamination are considered in the present study. The results show that the degrees of leading edge erosion have great influence on the flow separation, tangential force coefficient, normal force coefficient as well as the power output of the wind turbine. Leading edge erosion has the greatest impact on aerodynamics of the wind turbine at $15 \mathrm{~m} / \mathrm{s}$, where the maximum loss in the power output can reach up to $73.26 \%$.

Keywords: wind turbine, leading edge erosion, flow field structure, aerodynamic performance
\end{abstract}

\section{Introduction}

Wind energy, as a clean and renewable energy, is among the main directions in global new energy development and utilization. At present, the global total installed wind power capacity has exceeded $743 \mathrm{GW}$, and investment in wind power construction will continue to increase (IEA, 2020; GWEC, 2021). Restricted by wind resources and construction sites, wind farms are usually constructed in places with harsh climate and environment. As always operates in rain, snow, hail, sandstorm, salinity, and strong ultraviolet radiation conditions, erosion problems are easily to be observed at the leading edge of wind turbine blades, which greatly affects the operating safety and efficiency of the wind turbine (Ge et al., 2019). Zhang et al. (2015) pointed out that raindrops destroy the blade protective layer like bullets. Conan et al. (2012) found that sand particles carried by the airflow can also produce damage and erosion issues on the surface of wind turbine blades. In addition, tears and scratches on the surface of blade during manufacturing, transportation and installation may also be the root of erosion (Rempel, 2012). Wind power companies have found that leading edge erosion can be detected only after two years of installation. the first is small pits and cracks emerging on the blade surface (Sareen et al., 2012). After running for three years, numerous pits appear on the surface of the blade, and this is particularly serious near the leading edge of the upwind section of the blade, causing a drastic increase of the resistance of the wind turbine during operation (Bech et al., 2018). With a operation time increase, through-cavitation pits may appear on the surface of the blade, resulting in continuous erosion, maybe gouges or delamination, which also leads to a decrease in the lightning protection index of the blade. If no repair measures are taken, the erosion area and

\footnotetext{
${ }^{1}$ Presented paper from the 4th International Conference on Material Strength and Applied Mechanics, MSAM 2021
} 
depth will be further increased, causing large-areas erosion or delamination, which will severely restrict the power generation efficiency and safety operation of the wind turbine (Green New Wind Power Equipment Maintenance Co. Ltd., 2009; Sareen et al., 2014). The leading edge appearances of wind turbine blades after several years in operation are demonstrated in Fig. 1.

(a)

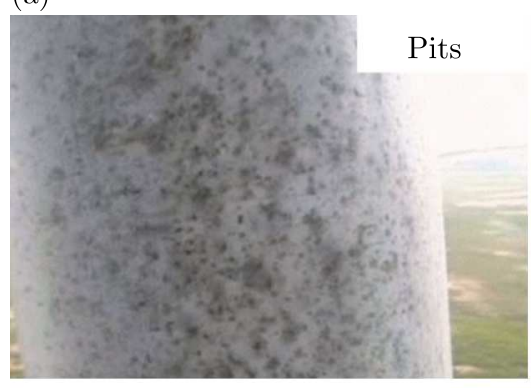

(d)

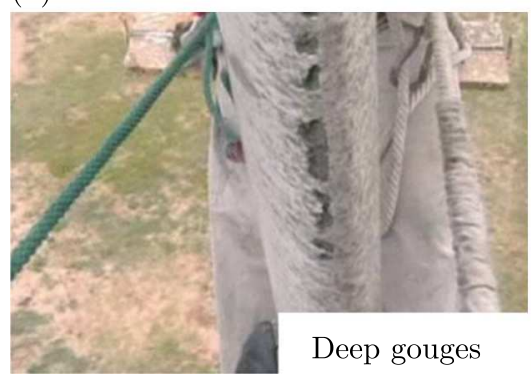

(b)

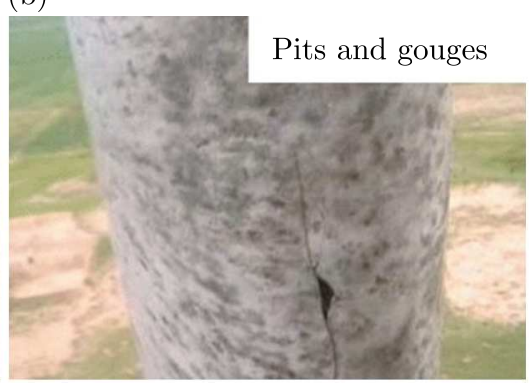

(e)

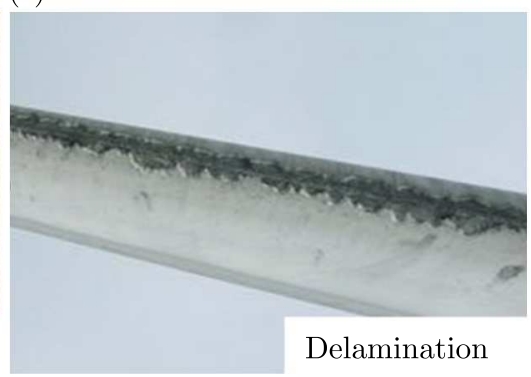

(c)

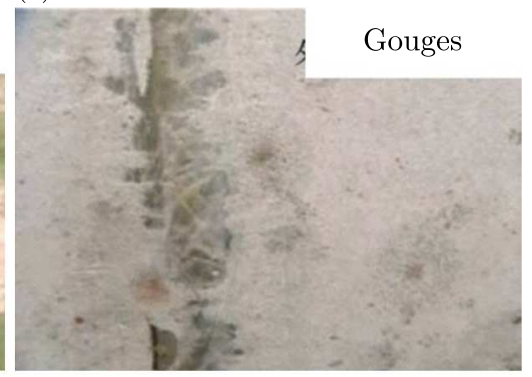

(f)

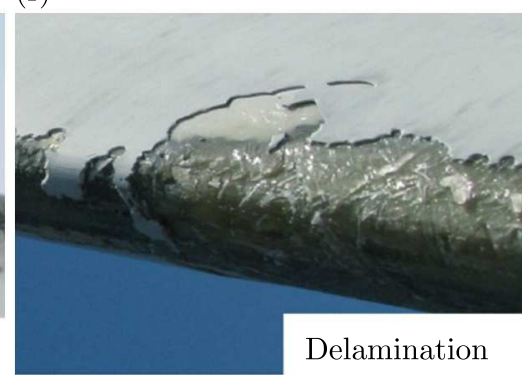

Fig. 1. Photographs of the eroded wind turbine blade after several years of operation (Rempel, 2012)

The blade is a key component for wind energy absorption and transformation, and its erosion at the leading edge has introduced severe problems to many wind power enterprises (Keegan et al., 2013). The performance and power generation efficiency of eroded wind turbines will reduce significantly, and finally affect the management plan and maintenance strategy made for a wind farm, which is closely related to economic benefits of the wind power enterprise (Sørensen, 2009). A 3M company found through field experiments that the power loss of a $1.5 \mathrm{MW}$ wind turbine with no leading edge protection could reach 20\%-30\% after five years of operation (Powell, 2011). Sareen et al. $(2012 ; 2014)$ showed through wind tunnel experiments that various leading edge erosion can increase the drag coefficient of DU $96-\mathrm{W}-180$ by $80 \%$ to $200 \%$, and in severe cases, it can cause a $25 \%$ loss in the annual power generation of $2.5 \mathrm{MW}$ wind turbines. Gharali and Johnson (2012) showed through numerical simulation that the leading edge defect can cause a lift coefficient decrease of $76 \%$ for S809 airfoil. Gaudern et al. (2014) investigated the erosion degrees of the Vestas wind turbine blade after five years of service and found that leading edge erosion reduces the lift-drag ratio of the airfoil by about $35 \%$ to $47 \%$. Ge et al. (2019) predicted the aerodynamic performance of the S809 airfoil under two kinds of leading edge defects, and found that the lift coefficient of the airfoil decreased significantly (by $35 \%$ to $61 \%$ ), and the corresponding drag coefficient increased by $131 \%$ to $217 \%$. Han et al. (2018) via simulations also found that leading edge erosion will cause the annual power generation of a $5 \mathrm{MW}$ wind turbine to decrease by $2 \%$ to $3.7 \%$. It can be concluded that the leading edge erosion has significant impact on aerodynamic characteristics and power output of a wind turbine. Furthermore, the maintenance of wind turbine blades is very expensive. According to the reports, the cost of wind turbines blade maintenance during their entire service life has reached $20 \%$ to $25 \%$ per kilowatt hour (kWh) (Mishnaevsky, 2019). Therefore, the understanding and prediction of the effect of the leading edge erosion on aerodynamic and flow field characteristics of the wind turbine blade is helpful to optimization of wind turbine design, safe operation, blade maintenance 
and management of wind farms, finally resulting in the economic growing of the wind power enterprise.

For the importance of understanding of the effects of leading edge erosion, researches on this topic have attracted an intense interest of scholars for the past few years (Herring et al., 2019; Ibrahim and Medraj, 2020). However, most studies were carried out with two-dimensional airfoils, such as Gharali and Johnson (2012), Wang (2017), Wang et al. (2016, 2017), Ge et al. (2019) conducted their research on the effects of leading edge erosion with S809 airfoil, which was greatly different from the three dimensional wind turbine model. A small amount of the research calculated the power loss of the wind turbine with the leading edge erosion, but the research was carried out with the BEM theory and the software of FAST or BLADED, such as the research of Han et al. (2018) and Sareen et al. (2012, 2014), which just obtained the values of power output but could not present a clear elaboration on the flow structures around the turbine blade. Therefore, a study on the impacts of leading edge erosion on wind turbine aerodynamic characteristics and the flow field structure under three-dimensional conditions is needed, which will provide better insights into the power loss and flow mechanism of the leading edge eroded wind turbine. The results of the present study can provide references for design, optimization, maintenance and management of the wind turbine blade.

\section{Geometrical model and computational method}

\subsection{Leading edge eroded configuration of Phase VI rotor}

In the present work, Phase VI wind turbine from National Renewable Energy Laboratory is selected to conduct the research for its detailed and abundant experimental data. The turbine is two-bladed with a diameter of $10.058 \mathrm{~m}$, and the blade configuration is made up of S809 aerofoil, detailed specifications of the turbine model can be looked up in (Mo and Lee, 2012) and (Giguere and Selig, 1999). The upwind direction without the yaw of turbine Phase VI is studied, and the influence of the tower is ignored. The current research reported and photographed of wind turbines by field observation showed that the erosion of the wind turbine blade leading edge may undergo three stages: pits, gouges and delamination.

It has been reported that the pits are commonly small and always spanned over the first $10 \% c$ of the blade (Sareen et al., 2012, 2014). Gaudern (2014) described the pit depth always ranging from $0.1 \mathrm{~mm}$ to $1.2 \mathrm{~mm}$ in a region that varies from $3 \% c$ to $8 \% c$, where $c$ is the chord length of the aerofoil. In the experimental study of Sareen et al. $(2012,2014)$, the pits and delamination were set to distribute in the first $10 \% c$ of the suction surface and $13 \% c$ of the pressure surface of the blade leading edge. To shed light on the impacts of the leading edge erosion on the aerodynamic characteristics, limit streamline and flow field structures of rhe wind turbine blade, the pits and three levels of delamination are set within the $10 \% c$ of the surface of the wind turbine blade. A schematic plot of the eroded leading edge configuration is shown in Fig. 2. All the results are compared with those of Phase VI turbine blade with no erosion, and the specific erosion parameters are illustrated in Table 1.

Table 1. Erosion parameters of Phase VI wind turbine blades

\begin{tabular}{|c|c|}
\hline Erosion treatments & Leading edge characteristics \\
\hline \hline Smooth blade & No erosion \\
\hline Erosion 1 & Pits erosion, $h=1 \mathrm{~mm}, x=10 \% c$ \\
\hline Erosion 2 & Delamination, $h=0.5 \mathrm{~mm}, x=10 \% c$ \\
\hline Erosion 3 & Delamination, $h=1 \mathrm{~mm}, x=10 \% c$ \\
\hline Erosion 4 & Delamination, $h=3 \mathrm{~mm}, x=10 \% c$ \\
\hline
\end{tabular}




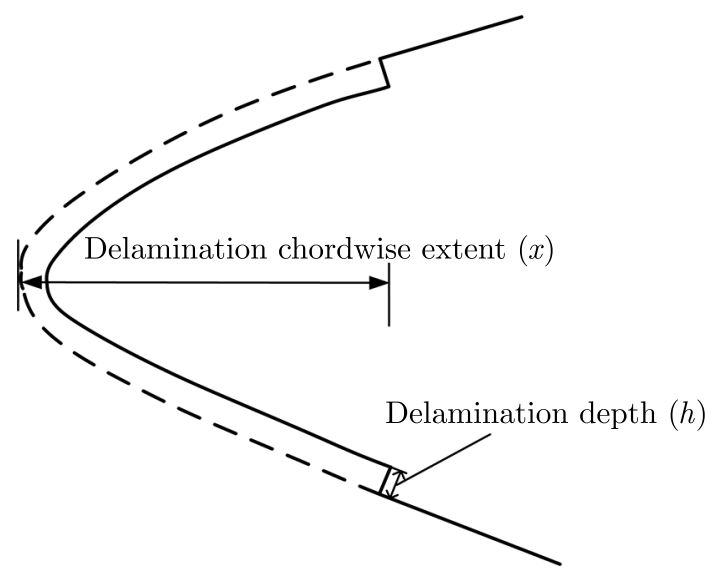

Fig. 2. Leading edge erosion configuration of Phase VI turbine blade

\subsection{Computational field and mesh generation}

An appropriate computational field is not only significant in obtaining accurate results but also very important in eliminating far-field boundary effects. In the present study, to compare the simulated results with those obtained from wind tunnel experiments (Simms et al., 2001), the wind turbine rotor is set at a height of $12.2 \mathrm{~m}$ from the ground, which is the same height of the rotor in the wind tunnel experiments. Meanwhile, the computational domain used in the simulation is similar to that of the experimental field, which consists of a semi-cylinder and a rectangular solid. The radius of the semi-cylinder is $50 \mathrm{~m}(10 R)$ and the height of the rectangular solid is $12.2 \mathrm{~m}$, and the total length of the domain is $82 \mathrm{~m}(16 R)$. The wind turbine is placed at $21 \mathrm{~m}$ (about $4 R$ ) from the inlet, as is demonstrated in Fig, 3.

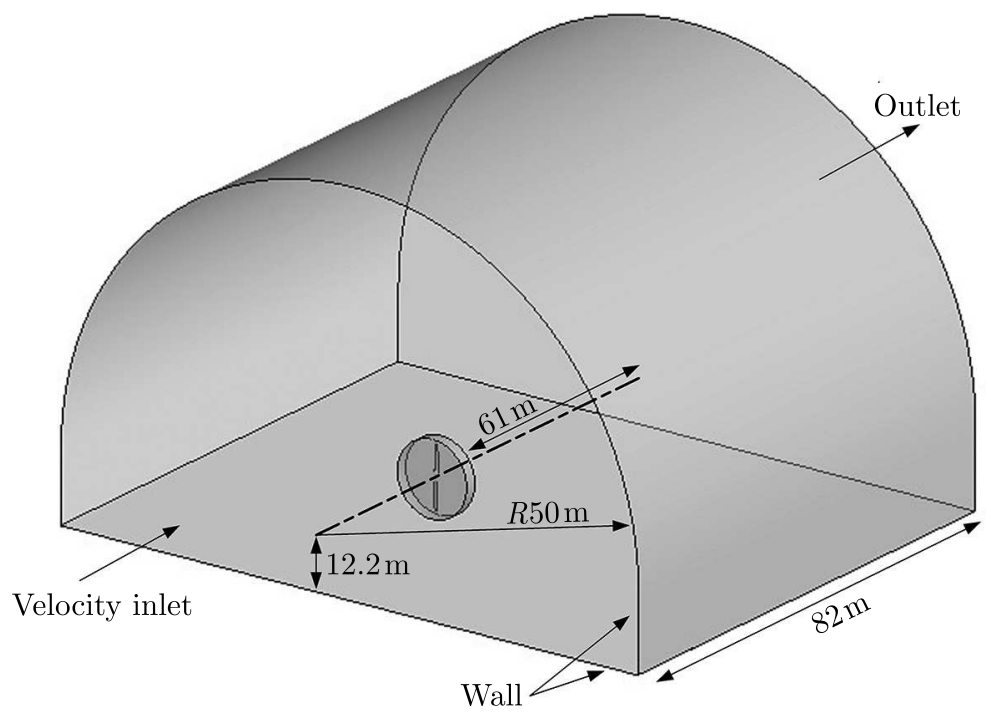

Fig. 3. Computational domain and boundary conditions

The numerical mesh is constructed with ICEM and structured, unstructured, and prism grids are combined. There are 7042700 grids arranged in the rotating domain, and about 3949900 grids are distributed in the static domain, and the total grid number is about 10 million. To catch the surface streamline and flow characteristics around the blade and meet the requirements of $y^{+} \approx 1$ in the turbulence model of SST $k-\omega, 40$ layers finer grids are added in the boundary layer of the blade surface, and the first cell height is approximately $5 \cdot 10^{-5} \mathrm{~m}$, no wall function 
is used in the simulation. The structured mesh around the blade and boundary layer and the prism mesh in the rotation domain are shown in Fig. 4.

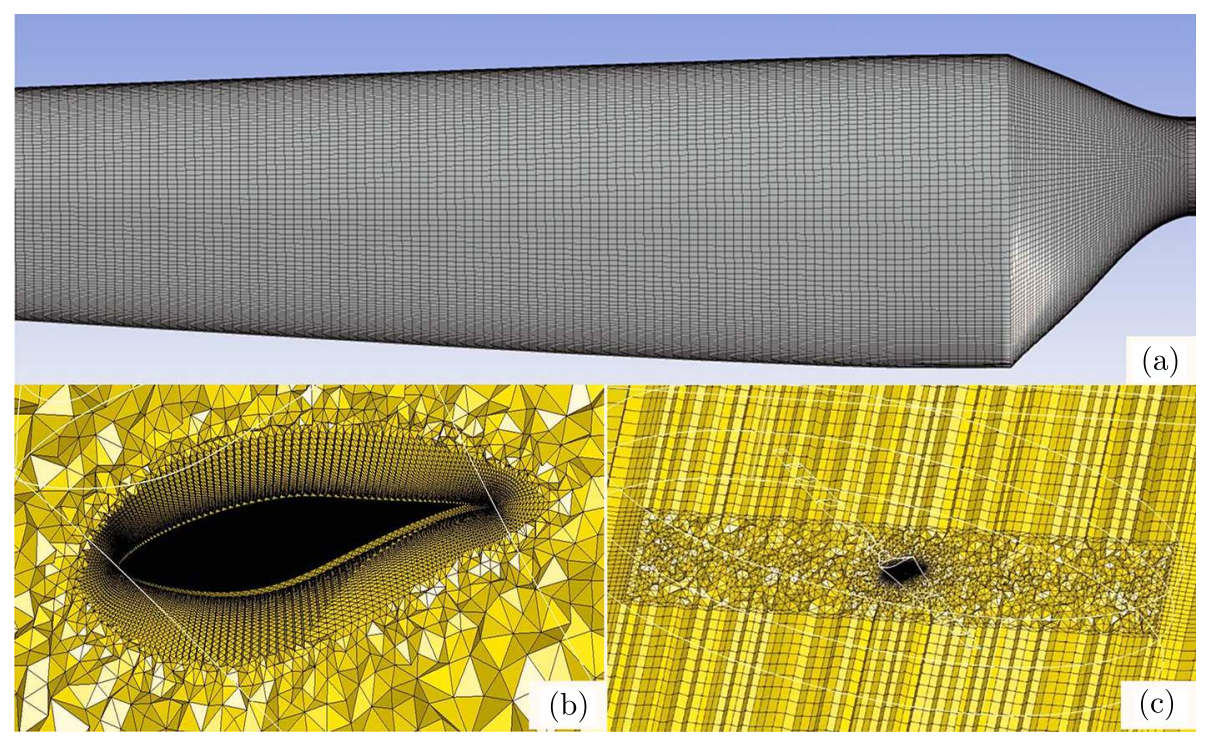

Fig. 4. Local grids near the blade in the boundary layer and rotating domain: (a) grids near the blade surface; (b) boundary layer grids; (c) hybrid mesh in the rotating domain

\subsection{Turbulence model and computational conditions}

Phase VI wind turbine is stall-regulated and the power output is limited due to the blade stall. The stall-regulated regime is a great difficulty in the CFD simulation to predict the stall initiation accurately. ANSYS Fluent 15.0 (Ansys, Inc.) is adopted in this study as it includes the well-known RANS equation and various turbulent models. The SST $k-\omega$ turbulence model is adopted throughout the study for its excellent results in the catching of flow separation and flow field details in 2D and 3D wind turbine blade simulation (Wang et al., 2016; Mo and Lee, 2012). This turbulence model effectively combines the advantages of the $k$ - $\omega$ model in the far field with the robust and accurate of $k-\omega$ model in the near-wall region (Menter, 1994). SIMPLE algorithm and a segregated implicit solver are adopted to couple the momentum and pressure equations. In addition, the MRF rotating frame is used for simulating rotation of the wind turbine. A second order scheme is adopted for discretization, and a residual of $10^{-6}$ is set for convergence.

In the calculation, a uniform velocity inlet and free flow boundary condition are used for the inlet and outlet, respectively. The non-sliding wall surface condition is adopted for the blade surface and ground. The sliding surface is adopted for the wind turbine surface, and the interface boundary condition is adopted for the interface between the rotating and static zone. Similarly to the blind comparison test, only the upwind and non-yawed turbine configurations have been used in the current analyses. The rotor is rotating at a constant speed of $72 \mathrm{rpm}$. The blade is no coning and has a pitch of 3 degree. The input parameters for computational cases are shown in Table 2.

\subsection{Numerical validation}

Figure 5 illustrates the simulation and experimental results of the pressure coefficients at cross sections $r / R=0.3, r / R=0.47, r / R=0.63, r / R=0.8$ and $r / R=0.95$ of the blade, respectively. The simulation is conducted at an incoming wind speed of $10 \mathrm{~m} / \mathrm{s}$, where $r$ is the position of cross section along the blade span, and $R$ is the radius of the wind turbine. In Fig. 5, $x$ represents the position of the calculation point in the chord direction of the cross section, and 
Table 2. Computational parameters for non-yawed, upwind Phase VI rotor

\begin{tabular}{|c|c|c|c|c|c|}
\hline Cases & $\begin{array}{c}\text { Wind tunnel } \\
\text { air temp } \\
{\left[{ }^{\circ} \mathrm{C}\right]}\end{array}$ & $\begin{array}{c}\text { Rotational } \\
\text { speed } \\
{[\mathrm{rpm}]}\end{array}$ & $\begin{array}{c}\text { Wind } \\
\text { speed } \\
\mathrm{m} / \mathrm{s}]\end{array}$ & $\begin{array}{c}\text { Air } \\
\text { density } \\
{\left[\mathrm{kg} / \mathrm{m}^{3}\right]}\end{array}$ & $\begin{array}{c}\text { Dynamic viscosity } \\
\text { coefficient } \\
\times 10^{5}[\mathrm{~kg} /(\mathrm{ms})]\end{array}$ \\
\hline \hline S0700000 & 11.1 & 71.9 & 7 & 1.246 & 1.769 \\
\hline S1000000 & 11.0 & 72.1 & 10 & 1.246 & 1.769 \\
\hline S1500000 & 14.2 & 72.1 & 15.1 & 1.224 & 1.784 \\
\hline S2000000 & 14.5 & 72.0 & 20.1 & 1.221 & 1.786 \\
\hline
\end{tabular}

$c$ is the chord length of the section. The dimensionless pressure coefficient of the blade section is calculated as

$$
C_{p}=\frac{P-P_{\infty}}{\frac{1}{2} \rho\left[U_{\infty}^{2}+(\omega r)^{2}\right]}
$$

(a)

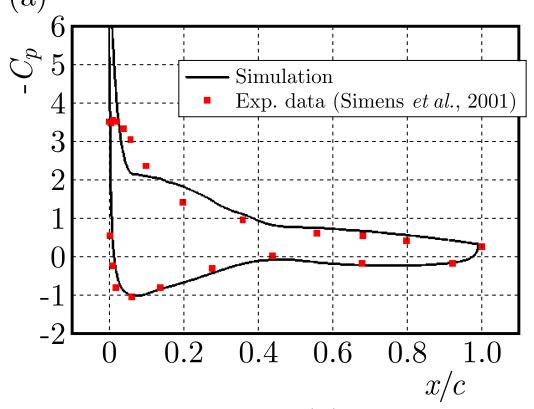

(d) (b)

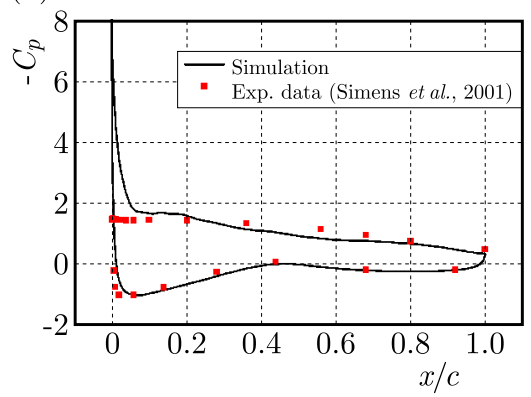

(c)

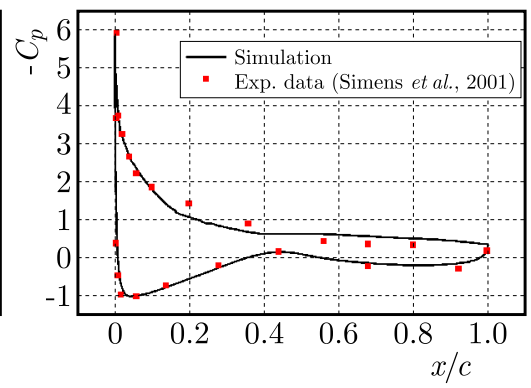

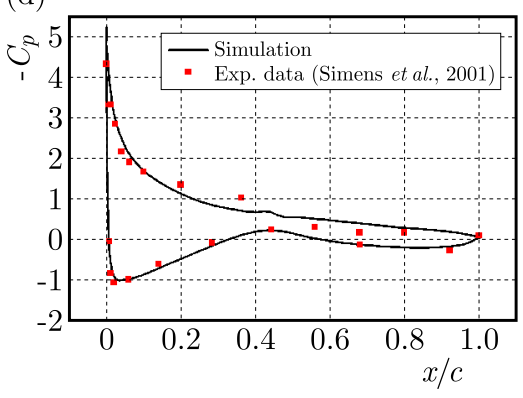

(e)

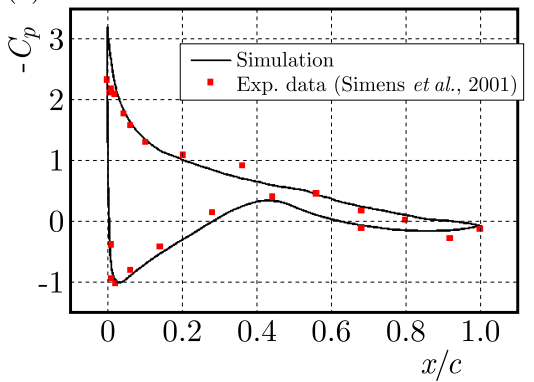

Fig. 5. Simulated and experimental pressure distributions of the blade at $10 \mathrm{~m} / \mathrm{s}$ wind velocity

As is shown in Fig. 5, the pressure coefficient calculated in the current study agrees well with the experimental results on the whole. For the section of $r / R=0.3$, the calculated value is consistent with the experimental result except for a little underestimate around the leading edge of the suction surface. At the position $r / R=0.47$, the pressure of the blade suction surface is still underestimated within the area of $10 \% c$ of the leading edge, resulting in a simulation error of $10.2 \%$ emergence at the point of $0.055 c$ of the cross section. This is mainly caused by high angles of attack and flow separation at the inside of the blade. For the position $r / R=0.63$, the simulated pressure coefficient at the blade leading edge is in good accordance with the experimental result, only a small error can be observed at $0.2 c$ of the blade suction surface. Similar results also appear at the sections of $r / R=0.8$ and $r / R=0.95$, with the maximum error being $15.6 \%$. This is mainly due to different linear velocity and angle of attack corresponding to the incoming flow at each cross section of the blade, which results in larger flow separation and finally cause a not satisfied simulation error at the root of the blade. At the same time, the three-dimensional flow effect of the blade also has a certain impact on the simulation accuracy. 
However, in general, the simulation results obtained with this turbulence model in the present study are close to the experimental results, and the numerical simulation method used can capture the most flow characteristics around the blades. The results obtained can be used for further analysis.

\section{Results and analysis}

\subsection{Effects on surface flow characteristics}

Figure 6 shows the surface streamline distributions on the suction surfaces of blades with different degrees of erosion at the incoming velocity of $7 \mathrm{~m} / \mathrm{s}, 10 \mathrm{~m} / \mathrm{s}, 15 \mathrm{~m} / \mathrm{s}$ and $20 \mathrm{~m} / \mathrm{s}$, respectively. Because differences in the limiting streamlines of the blade pressure surface among

(a)

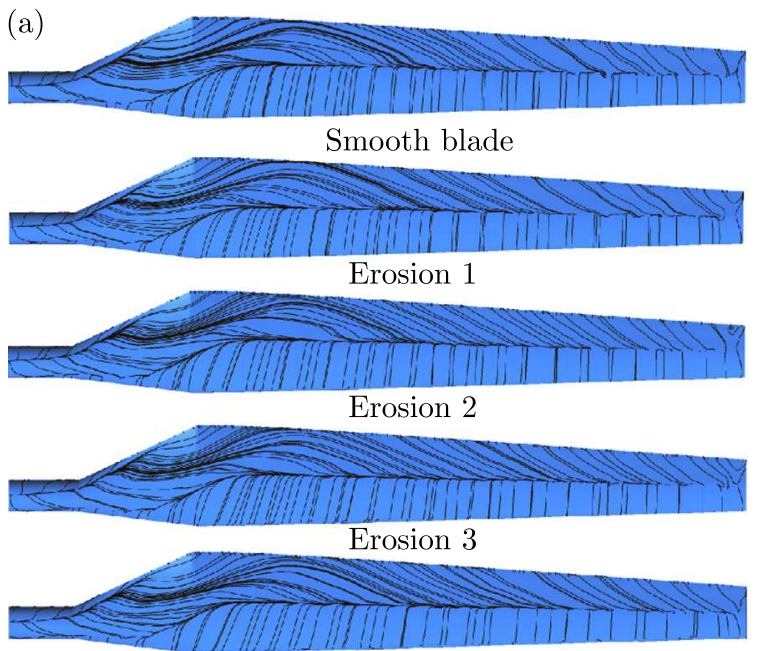

Erosion 4

(c)

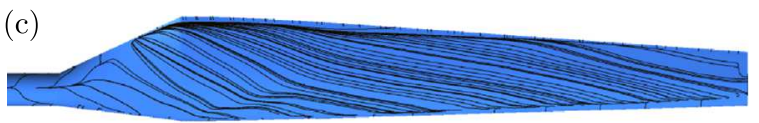

Smooth blade

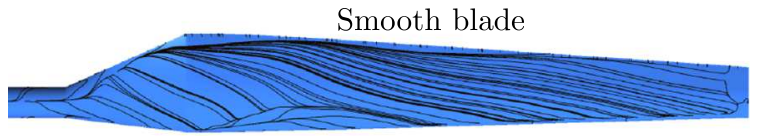

Erosion 1

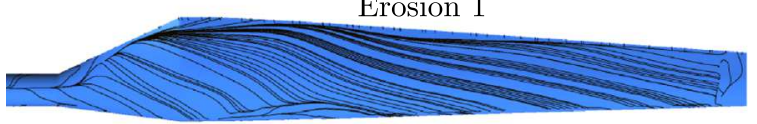

Erosion 2

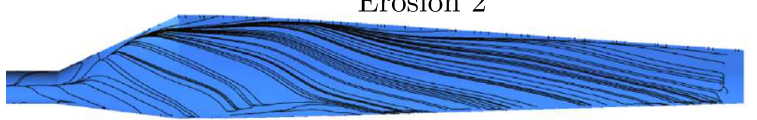

Erosion 3

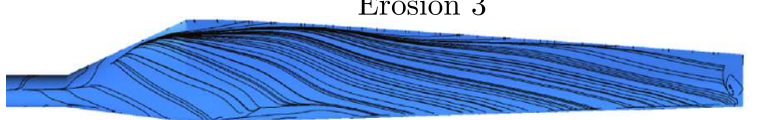

Erosion 4

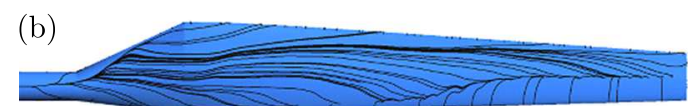

Smooth blade

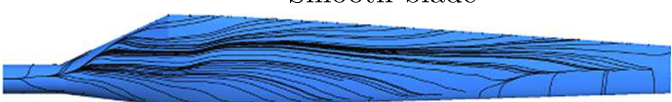

Erosion 1

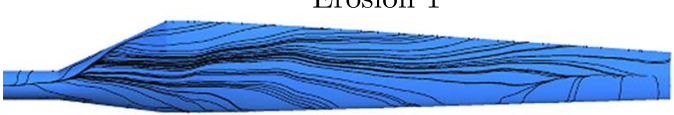

Erosion 2

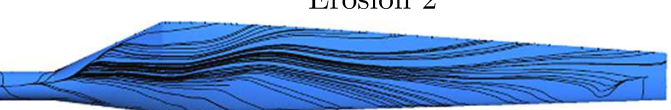

Erosion 3

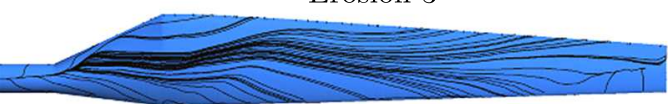

Erosion 4

(d)

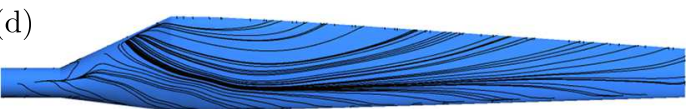

Smooth blade

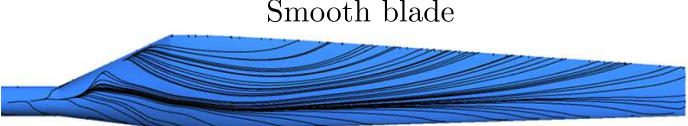

Erosion 1

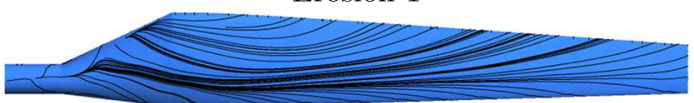

Erosion 2

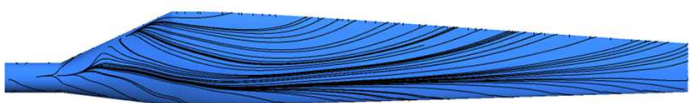

Erosion 3

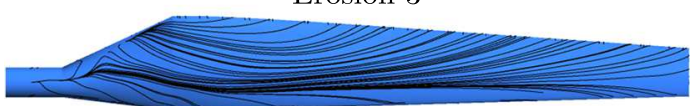

Erosion 4

Fig. 6. Blade surface streamlines under various wind speeds and leading edge erosion: (a) $7 \mathrm{~m} / \mathrm{s}$, (b) $10 \mathrm{~m} / \mathrm{s}$, (c) $15 \mathrm{~m} / \mathrm{s}$, (d) $20 \mathrm{~m} / \mathrm{s}$

various cases are small, only the suction surface is analyzed here. It can be seen from Fig. 6 that the surface limiting streamlines of turbine blades with various degrees of erosion are generally the same at an incoming wind velocity of $7 \mathrm{~m} / \mathrm{s}$. Meanwhile, the flow separation points are all in the middle position of the surface of the cross section. For the area near the leading edge, 
the attached flow can be observed for all investigated cases, while the spanwise separated flow cover the other half section, from the middle to the trailing edge. In contrast, the spanwise flow spans over the most region of the suction surface of the blade when the wind velocity is $10 \mathrm{~m} / \mathrm{s}$, which is due to the occurrence of stall couples with the action of the centrifugal force. There are obvious differences in the surface limiting streamlines for blades with different levels of leading edge erosion, especially in the outboard of the blade. As the leading edge erosion aggravates, the area of the separated flow gradually increases. When the erosion depth increases to $3 \mathrm{~mm}$, the spanwise separated flow almost covers the entire blade, which illustrates that the degree of leading edge erosion significantly affects the position of the flow separation point on the blade surface. In other words, as the degree of erosion increases, the larger the flow separation zone and the closer of the separation point is to the leading edge. When the wind speed accelerates to $15 \mathrm{~m} / \mathrm{s}$, only a small range of the attached flow appears near the leading edge tips of the smooth and pitted blades, while the spanwise flow covers the entire suction surfaces of the three levels of delaminated blades. For the inlet wind velocity of $20 \mathrm{~m} / \mathrm{s}$, the surfaces of the five studied cases are all covered by the spanwise flow, the differences of surface limiting streamlines among different erosion levels are generally the same, indicating that leading edge erosion has little influence on surface flow characteristics of the wind turbine blade under a high wind speed.

\subsection{Effects on flow field structures}

Figure 7 displays the flow field structure of the circumfluence at different cross-sections along the blade at the wind velocities of $10 \mathrm{~m} / \mathrm{s}$ and $20 \mathrm{~m} / \mathrm{s}$. The blade is subjected to various degrees of erosion and the results are compared with those of the smooth blade. As is shown in Fig. 7a, flow separations appear at all cross-sectional positions $(0.30 R, 0.47 R, 0.63 R, 0.80 R, 0.95 R)$ for both smooth and eroded blades when the wind speed is $10 \mathrm{~m} / \mathrm{s}$. However, the flow separation

(a)

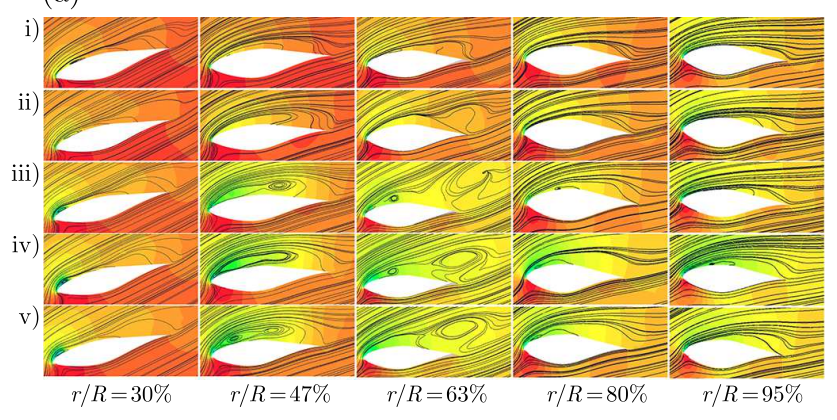

(b)

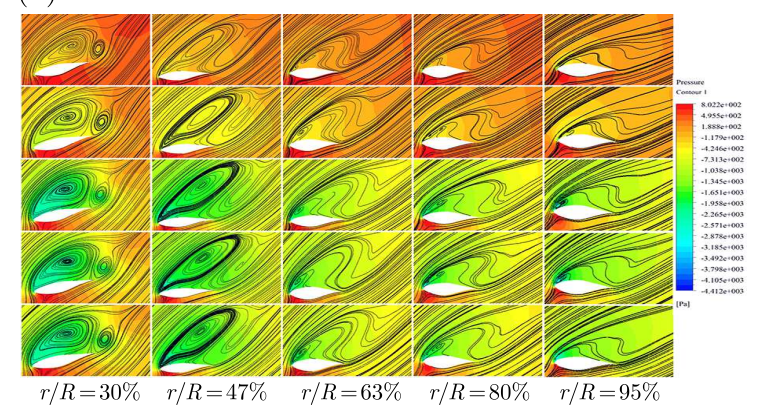

Fig. 7. Flow structures around the blade section at the wind speed of (a) $7 \mathrm{~m} / \mathrm{s}$ and (b) $20 \mathrm{~m} / \mathrm{s}$ : i) smooth blade, ii) erosion 1, iii) erosion 2, iv) erosion $3, \mathrm{v}$ ) erosion 4

characteristics are different for blades with various degrees of erosion. At the cross section $0.30 R$, a small flow separation region appears on the suction surface near the leading edge of all eroded blades, and the separation zone is very small and can be considered as a leading edge separation bubble. The size of this separation bubble grows with the increase of erosion degree. This is mainly due to the angle of attack at the inside of the blade which is relatively high, and the fact that the stagnation point at the lower surface has a little distance from the front edge of the blade. When the airflow moves from the stagnation point to the upper surface, it needs to bypass a "thin wing" formed by leading edge delamination and, therefore, the lowest pressure point on the upper surface will be very close to the leading edge. There exists a long adverse pressure gradient region following the lowest pressure point, which will cause the flow separation and finally form the leading edge separation bubble. As the blade cross section moves from the blade root to tip, the angle of attack gradually decreases and the area of the flow separation 
zone at the blade tip reduces and gradually disappears, with flow separation only existing near the tailing edge. Comparing the flow field structure around the same cross-section of blades with different degrees of erosion, it can be found that the flow separation phenomena become more obvious with the increase in erosion levels, and the location of the separation point moves closer to the leading edge. In addition, the flow separation occurs earlier and the flow separation region is larger with the growing erosion degree, causing a more severe drop in the aerodynamic performance of the turbine.

As is shown in Fig. 7b, when the wind velocity increased to $20 \mathrm{~m} / \mathrm{s}$, all the eroded cases studied have notable flow separations around each cross section of the blade. The closer it is to the blade root, the closer the flow separation position is to the leading edge, and more similar the shape of the separation vortex is to a circle. As the cross section moves from the root to tip of blade, the thickness of the separation vortex gradually decreases and the size of the separation vortex becomes smaller. The effect of the separation vortex at the blade root is equivalent to increasing relative thickness of the aerofoil at the cross section and increasing shape resistance, resulting in a reduction of the turbine output of the blade. Comparing the streamlines at the same cross-sectional position of blades with different erosion degrees, one can see that the size of the separation vortex of all eroded blades is larger than that of the smooth blade. Meanwhile, the flow separation region of the three delaminated blades is slightly larger than that of the pitted rough one, as well as the pressure in the separation zone is lower for the delaminated blades. There is almost no difference in the flow field structure at the cross section $0.30 R, 0.47 R$, and $0.63 R$ among the 3 delaminated blades, which illustrates that the leading edge delamination thickness has little influence on the flow characteristics of the inside of blades when they are in deep stall. In addition, the cross-sectional flow field structures of blades with $0.5 \mathrm{~mm}$ and $1 \mathrm{~mm}$ delamination thickness are similar to each other for the flow field structure at $0.8 R$ and $0.95 R$ cross-sections, while the size of the separation vortex of the $3 \mathrm{~mm}$ delaminated blade is significantly larger, as well the angle between the streamline of the outer edge of the separation vortex and the chord line is larger than the former two cases. This indicates that the wind turbine with $3 \mathrm{~mm}$ delamination blade suffers a more severe reduction in the aerodynamic performance.

\subsection{Effects on section normal and tangential forces}

Figure 8 shows the distribution of the normal force coefficient $C_{n}$ at each cross section along the spanwise of the blade with different levels of leading edge erosion. It can be observed that there is almost no difference in the normal force coefficient $C_{n}$ at the position $r / R=30 \%$ for blades with different degrees of erosion under a low wind speed of $7 \mathrm{~m} / \mathrm{s}$. However, the differences caused by leading edge erosion are significant for other cross sections at this wind speed. The result illustrates that leading edge erosion has little impact on the normal force for sections at the inside of the blade at low inflow speeds. When the wind velocity grows up to $10 \mathrm{~m} / \mathrm{s}$ and $15 \mathrm{~m} / \mathrm{s}$, the normal force coefficient of the eroded blade is greater than that of the smooth blade at the section $r / R=47 \%$, while smaller than the smooth blade at the other sections, the normal force coefficient has a peak value at this section. When the inflow velocity increases to $20 \mathrm{~m} / \mathrm{s}$, the peak value section moves forward to the position of $r / R=63 \%$, indicating that leading edge erosion increases the aerodynamic loading in the middle of the blade. Making a comparison among the normal force coefficients of the same cross section under different wind speeds, the results shows that the impacts of leading edge erosion on the normal force coefficient $C_{n}$ gradually decreases with an increase in the wind speed. Especially, values of the normal force coefficient $C_{n}$ of each cross section change weakly at different wind speeds for the blade with various levels delamination. This is because the increase in the wind speed causes various degrees of stall at each cross section of the blade, and the change in the degree of leading edge delamination has less 
influence on the flow field structure around the blade which, in turn, causes a smaller influence on the normal force coefficient $C_{n}$ after the blade is install.

(a)

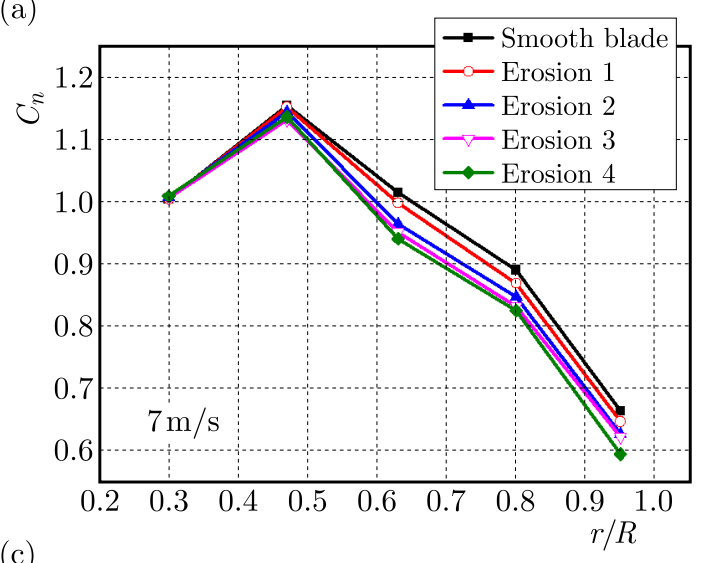

c)

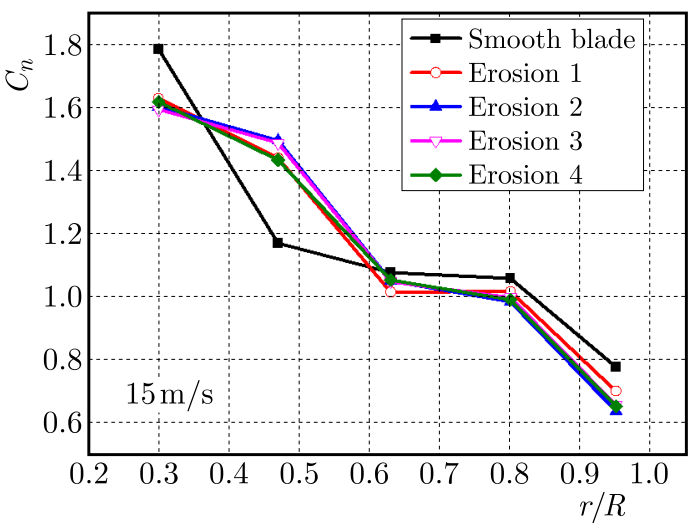

(b)

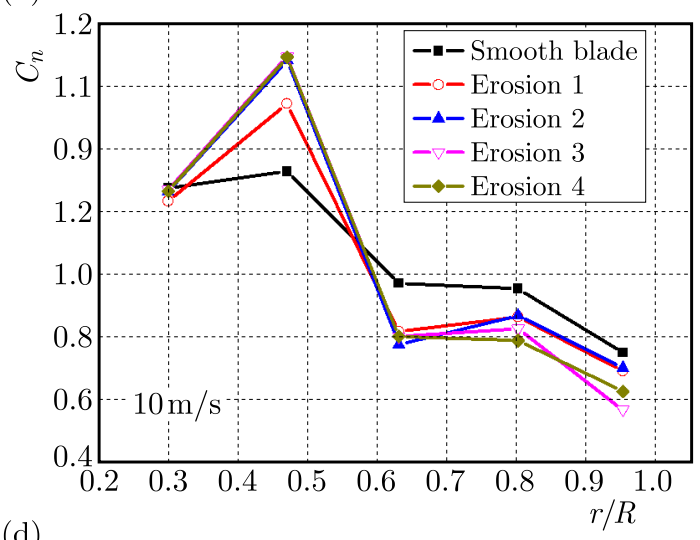

d)

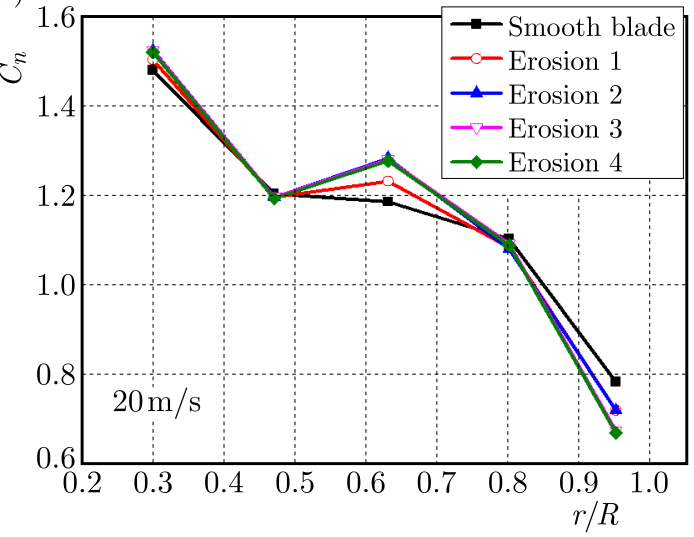

Fig. 8. Distribution of the normal force coefficient $C_{n}$ along the blade under different wind velocities

Figure 9 illustrates the distribution of the cross-sectional tangential force coefficient $C_{t}$ along the spans of blades with various degrees of leading edge erosion under different wind speeds. It illustrates that the differences of the section tangential force coefficient $C_{t}$ are not obvious among all investigated cases at the relative low wind speed of $7 \mathrm{~m} / \mathrm{s}$, especially at the position $r / R=0.3 \%$. The trend of the tangential force coefficient among different cases is in line with the behavior of the normal force coefficient. However, with an increase in the wind speed, the tangential force coefficient $C_{t}$ of the blades significantly decreases after the leading edge is eroded. When the wind speed reaches $20 \mathrm{~m} / \mathrm{s}$, the tangential force of the entire blade is significantly lower than that of the smooth blade. Moreover, the tangential force coefficients of the three level delaminated blades are almost the same along the entire blade span, and the values of which are much lower than those of the pitted blade. This is because the entire blade goes into stall as the wind speed accelerates, the effect of the erosion depth at the leading edge on flow characteristics around the blade decreases, which is consistent with the conclusion in Section 3.2. Comparing the tangential force coefficients at different positions of the blade, it shows that the tangential force coefficient $C_{t}$ of the outboard section of the blade reduces more significantly than the inside section of the blade. That is, relative to the sections near the blade root, the effect of delamination on the tangential force coefficient $C_{t}$ is greater than that in sections of near the blade tip. This may be caused by the high relative speed and output in the outboard of the blade combined with the strong three-dimensional flow effect of the tip, finally leading to a higher decline in the aerodynamic force. 
(a)

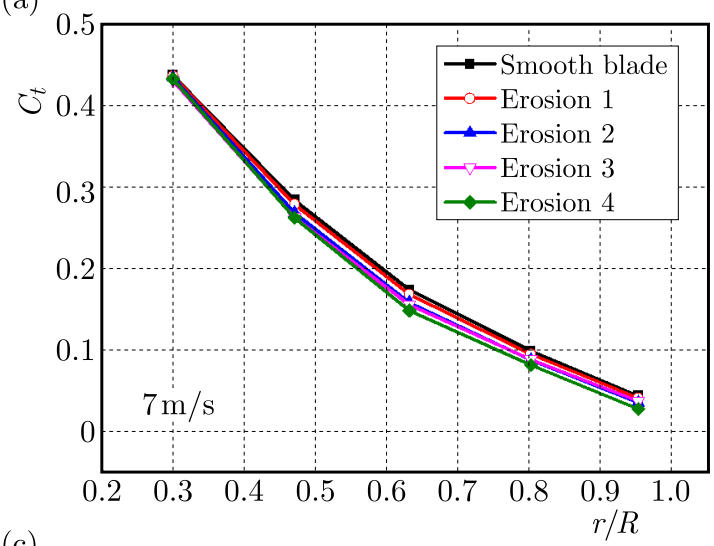

(c)

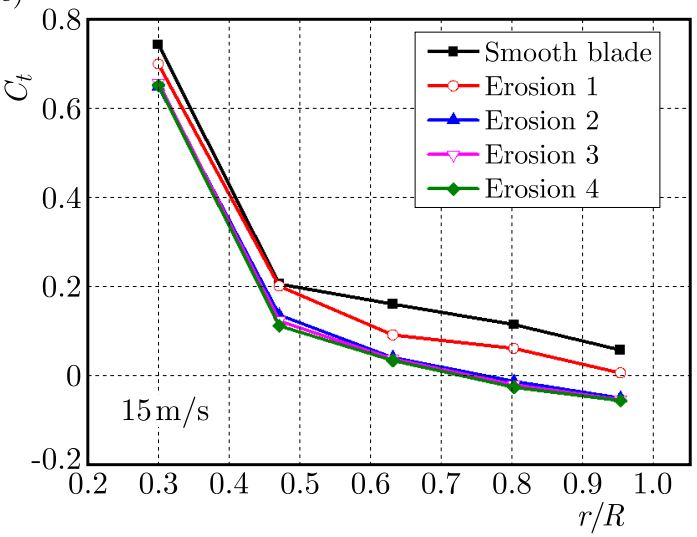

(b)

(d)
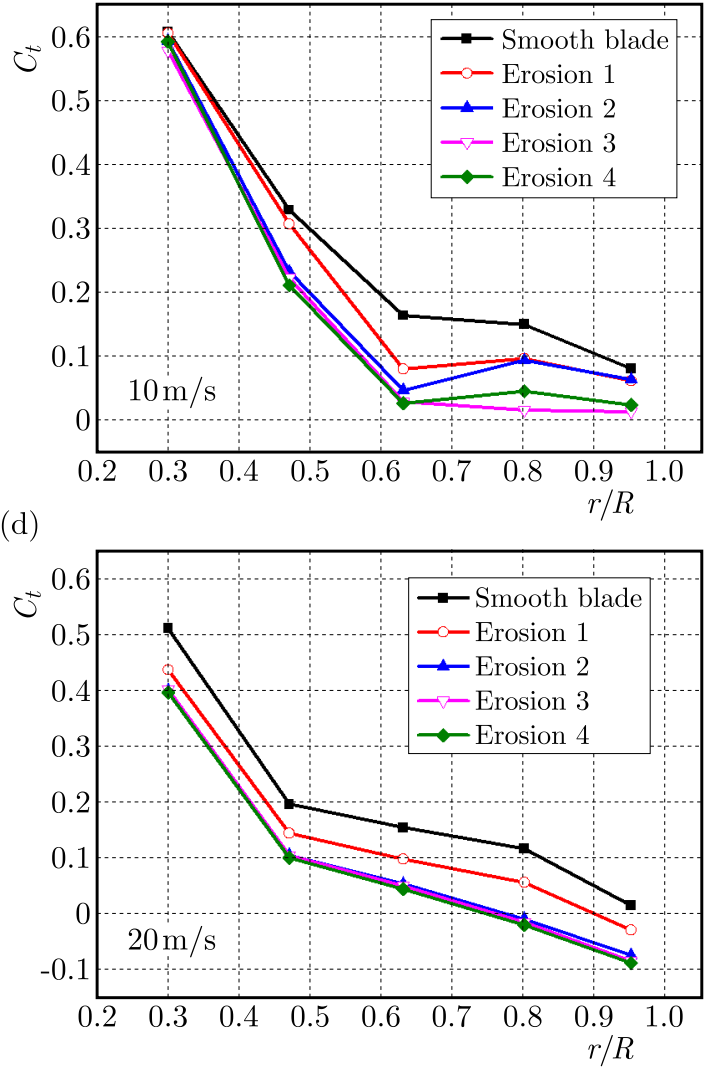

Fig. 9. Distribution of rgw tangential-force coefficient $C_{t}$ along the blade under various wind speeds

\subsection{Effects on rotor torque and thrust}

Figure 10 presents the variation trend of rotor torque and thrust with the incoming wind velocity for blades with different degrees of erosion. It can be seen that the rotor thrust decreases as the degree of erosion increases. Meanwhile, with the accelerating wind speed, the reduction of the rotor thrust under different degrees of erosion first increases and then decreases, which is agreement with the variation law of the normal force coefficient $C_{n}$ along the blade radius. When the wind speed increases to $20 \mathrm{~m} / \mathrm{s}$, the leading edge erosion presents a very small effect on the rotor thrust. That is, the impacts of leading edge erosion degrees on the rotor thrust are reduced under high wind speeds. On the contrary, the rotor torque is significantly influenced by the leading edge erosion, and the effect grows with acceleration of the wind speed. Compared with the smooth blade, the rotor torque decreases as the erosion degree gets severe, which is especially obvious at higher wind speeds. For a small incoming wind velocity of $7 \mathrm{~m} / \mathrm{s}$, the influence of erosion on the rotor torque is small, which is in accordance with the result of the surface streamline on the blade. Torque is the main parameter to characterize the output power of the rotor. Therefore, as the degree of erosion increases, the output power of the rotor decreases, and the power loss and economic losses increase. In addition, comparing the three types of blades with delamination, it illustrates that the power loss of the wind turbine caused by the delamination depth reduces with the acceleration in the wind speed, which is mainly due to the deep stall of the wind turbine blade at a high wind speed.

Table 3 presents the relative diminished power output of the wind turbine caused by different levels of leading edge erosion. For the inflow wind velocity of $7 \mathrm{~m} / \mathrm{s}$, the power loss of the wind turbine increases as the degree of erosion accelerates, the power decrease is just $2.95 \%$ for the pitting eroded the blade, while that is $13.69 \%$ for the rotor with the blade leading edge 
(a)

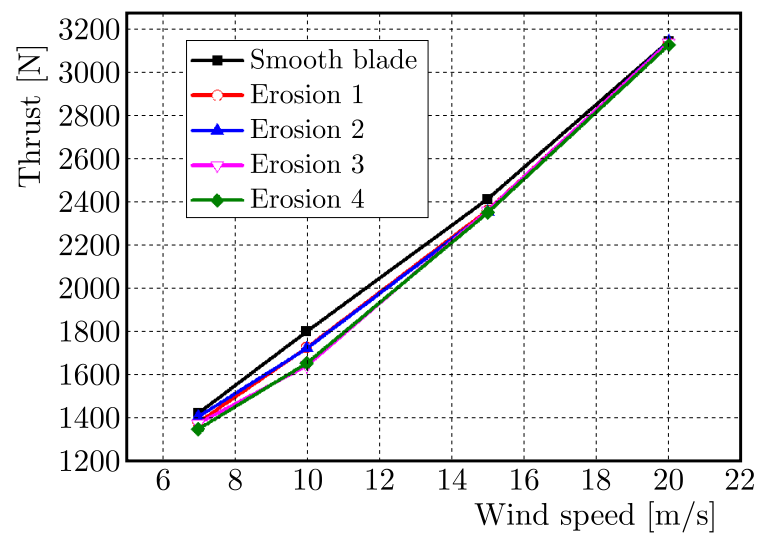

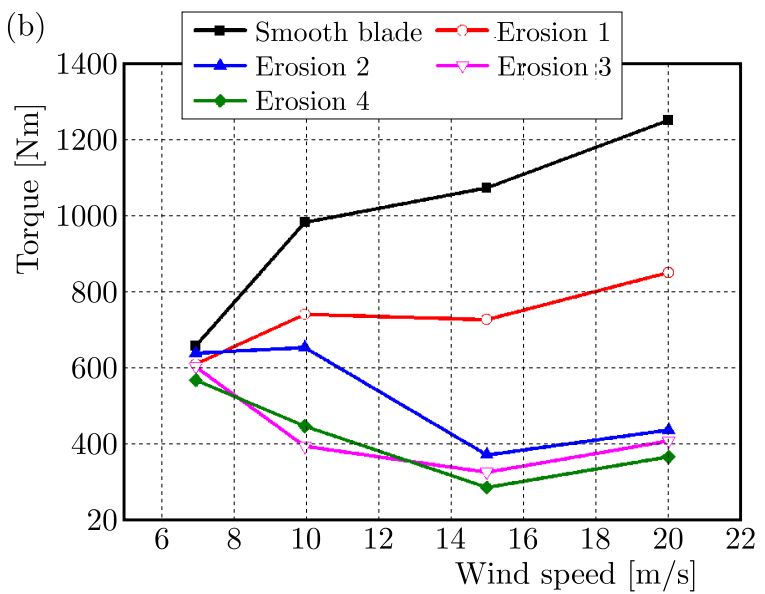

Fig. 10. Thrust and torque vary with wind speeds for various eroded Phase VI rotors

Table 3. Shaft power decrease for various eroded Phase VI rotors

\begin{tabular}{|c|c|c|c|c|}
\hline \multirow{2}{*}{$\begin{array}{c}\text { Wind speed } \\
{[\mathrm{m} / \mathrm{s}]}\end{array}$} & \multicolumn{4}{|c|}{ Shaft power decrease [\%] } \\
\cline { 2 - 5 } & Erosion 1 & Erosion 2 & Erosion 3 & Erosion 4 \\
\hline \hline 7 & 2.95 & 7.27 & 8.43 & 13.69 \\
\hline 10 & 24.58 & 33.47 & 53.82 & 58.91 \\
\hline 15 & 32.21 & 65.32 & 69.54 & 73.26 \\
\hline 20 & 31.94 & 65.01 & 67.31 & 70.64 \\
\hline
\end{tabular}

delamination depth of $3 \mathrm{~mm}$. When the incoming velocity reaches to $10 \mathrm{~m} / \mathrm{s}$, the power loss caused by erosion is about 4 to 8 times of that of the wind speed $7 \mathrm{~m} / \mathrm{s}$ for various degrees of the leading edge erosion. At this wind speed, the wind turbine power loss caused by pitting erosion is $24.58 \%$, while the maximum power loss caused by leading edge delamination is soaring to $58.91 \%$. The difference of power loss among the three level delaminated blade is about $20 \%$. When the incoming wind velocity increases to $15 \mathrm{~m} / \mathrm{s}$, the maximum reduction in wind turbine power caused by erosion can reach $73.26 \%$ when the delamination depth is $3 \mathrm{~mm}$, while the minimum power loss is $32.21 \%$ (pitting erosion). However, the difference in the rotor power loss caused by the three delamination cases is reduced to about $8 \%$. For the wind velocity of $20 \mathrm{~m} / \mathrm{s}$, the minimum power loss is $31.94 \%$ and the maximum drop is $70.64 \%$ for the investigated cases, and the differences among the three levels of delamination is only $5.63 \%$, which shows a weakening blade erosion effect as the wind velocity increases. Comparing the wind power loss caused by leading edge erosion at different wind velocities, it illustrates that the power drop ratio is the largest at $15 \mathrm{~m} / \mathrm{s}$, indicating that there may exist a peak wind speed for the impact of leading edge erosion on the power generation of the wind turbine. The impact of leading edge erosion on the power output increases first and then decreases with the increase in the wind speed.

\section{Discussions}

Wind energy is today a main source of electricity, and will continue playing a leading role in achieving carbon neutrality and climate goals. Leading edge erosion has been reported a common phenomenon for wind turbine blades, and shallow pits, surface gouges and delamination may appear near the leading edge after several years of operation. A fundamental understanding on the relation between various levels of erosion and a wind power decrease is crucial to improve reliability, predictability and management of wind farms. In the present study, as the erosion 
degree increases, the attached flow regime decreases, the leading edge separation bubble grows in size, and a severe decrease in shaft power is observed. At a small inflow velocity, the light erosion (pits erosion) can lead a $2.95 \%$ power loss, and severe erosion (delamination) can cause a power loss of $13.69 \%$. This value increases to $31.94 \%$ and $70.64 \%$ when the inflow velocity grows from $7 \mathrm{~m} / \mathrm{s}$ to $20 \mathrm{~m} / \mathrm{s}$. The results are basically in agreement with the results of Sareen et al. (2014) and Han et al. (2018). Sareen et al. (2012) showed that the drag coefficients increased by $6 \%$ to $500 \%$ for the leading edge degradation going from light to heavy, and the AEP loss estimated was about $3 \%$ to $5 \%$ for a small amount of leading edge erosion and could approach $25 \%$ for heavy erosion conditions, and the prediction was conducted with a $2.5 \mathrm{MW}$ wind turbine, and the Weibull inflow wind speed distribution $(k=2)$ was used. In Han's research, they pointed out that the light and severe leading edge erosion can cause a reduction of the minimum and maximum of the lift-drag ratio by $35.4 \%$ and $66.4 \%$. The annual energy production (AEP) losses were between $0.36 \mathrm{GWh}$ and $0.65 \mathrm{GWh}$, corresponding to a AEP loss of $2 \%$ to $3.7 \%$ at the inflow speed of $4 \mathrm{~m} / \mathrm{s}$ depending on the extent of leading edge damage. The AEP loss increases with the increase of inflow wind speed. The field experiments of 3M Company showed that the AEP loss of wind turbines without blade protection were about $20 \%$ to $30 \%$ after 5 years in operation (Powell, 2011). The AEP loss of Sareen et al. (2014) was derived from the outer part of the blade, and the delamination range was just $3 \% c$, and the wind speed in the study of Han et al. (2018) was $4 \mathrm{~m} / \mathrm{s}$, much smaller than the rated wind speed, while in the present study the delamination was $10 \% c$ and spanned over the whole blade, and the inflow velocity ranged from $7 \mathrm{~m} / \mathrm{s}$ to $20 \mathrm{~m} / \mathrm{s}$, and the power loss increased with the wind speed increase, so the power loss in the present study were a little greater than in the aforementioned study. However, delamination in the whole blade with a depth greater than $1 \mathrm{~mm}$ is an extreme event, but the pits erosion and light extent delamination can be observed in a real wind farm. Like in the case of Erosion 1 and Erosion 2, the predicted results are in accordance with the experimental results of 3M (Powell, 2011) and Sareen et al. (2014). Thus, the results in the present study can generally provide some basis for power generation prediction of a leading edge degraded wind turbine, which will be useful for blade design, optimization, protection and wind farm operation and management.

\section{Conclusions}

- Leading edge erosion has a significant impact on surface streamlines and flow structures around the blade. As the degree of erosion increases, the flow separation becomes more obvious, and the separation region is larger with the separation point being closer to the leading edge. In addition, erosion also induces the generation of leading edge separation bubbles on the upper surface of the inside of the blade, and the size of which increases with the degree of erosion. At higher wind speeds, the degree of erosion has little influence on flow separation and surface streamlines of the wind turbine blade.

- Leading edge erosion reduces the normal force coefficient in the whole blade at low wind speeds when compared with the smooth blade. However, with an increase in the wind speed, the leading edge erosion increases the normal force coefficient in the middle section of the blade, a peak value can be observed at the position of $r / R=0.47$ at the wind speeds of $10 \mathrm{~m} / \mathrm{s}$ and $15 \mathrm{~m} / \mathrm{s}$, while that is at $r / R=0.63$ for $20 \mathrm{~m} / \mathrm{s}$. The tangential force coefficient of the blade decreases with the aggravation of leading edge erosion. Meanwhile, the impacts of leading edge erosion, especially delamination, on the tangential force coefficient of the blade section gradually reduces with an increase in the wind velocity.

- The torque and thrust of the wind turbine decreases with the aggravation of leading edge erosion. At a fixed erosion depth, there exists a peak wind speed for the erosion effects on thrust and torque of the wind turbine. That is, the reduction ratios of the wind turbine torque and thrust increase first and then decrease with an increase in the wind speed. 
At $15 \mathrm{~m} / \mathrm{s}$, the impact of leading edge erosion on power is the most significant, with the maximum and minimum wind turbine power loss being $73.26 \%$ and $32.21 \%$, respectively. Furthermore, the differences among the three levels of leading edge delamination on the power loss of the wind turbine decrease with an increase in the wind speed.

\section{Acknowledgements}

This work was supported by the financial support from the National Natural Science Foundation of China (11902131), the Natural Science Foundation of Gansu Province (20JR5RA443), and Gansu Provincial Higher Education Science and Technology Achievements Transformation Project (2018D-04). The authors would like to thank D. Simms et al. from National Renewable Energy Laboratory for the details and data of the previous work, Xuyao Zhang from Lanzhou Jiaotong University for the help in current simulation.

\section{References}

1. Bech J.I., Hasager C.B., BaK C., 2018, Extending the life of wind turbine blade leading edges by reducing the tip speed during extreme precipitation events, Wind Energy Science, 3, 729748

2. Conan B., van Beeck J., Aubrun B.S., 2012, Sand erosion technique applied to wind resource assessment, Journal of Wind Engineering and Industrial Aerodynamics, 104-106, 322-329

3. Gaudern N., 2014, A practical study of the aerodynamic impact of wind turbine blade leading edge erosion, Journal of Physics Conference Series, 524, 012031

4. Ge M., Zhang H., Wu Y., Li Y., 2019, Effects of leading edge defects on aerodynamic performance of the S809 airfoil, Energy Conversion and Management, 195, 466-479

5. Gharali K., Johnson D.A., 2012, Numerical modeling of an s809 airfoil under dynamic stall, erosion and high reduced frequencies, Applied Energy, 93, 45-52

6. Giguere P., Selig M.S., 1999, Design of a Tapered and Twisted Blade for the NREL Combined Experiment Rotor, NREL/SR-500-26173, NREL, Golden, CO

7. GWEC, 2021, Global Wind Report 2020, https://gwec.net/global-wind-report-2021/

8. HAN W., Kim J., Kim B., 2018, Effects of contamination and erosion at the leading edge of blade tip airfoils on the annual energy production of wind turbines, Renewable Energy, 115, 817-823

9. Herring R., Dyer K., Martin F., Ward C., 2019, The increasing importance of leading edge erosion and a review of existing protection solutions, Renewable and Sustainable Energy Reviews, 115, 109382

10. Ibrahim M.E., Medraj M., 2020, Water droplet erosion of wind turbine blades: mechanics, testing, modeling and future perspectives, Materials, 13, 157

11. IEA, 2020, World Energy Investment 2020. International Energy Agency Publications, https:// www.iea.org/reports/world-energy-investment-2020

12. Keegan M.H., Nash D.H., Stack M., 2013, On erosion issues associated with the leading edge of wind turbine blades, Journal of Physics D: Applied Physics, 46, 3727-3748

13. Menter F.R., 1994, Two-equation eddy-viscosity turbulence models for engineering applications, AIAA Journal, 32, 1598-1605

14. Mishnaevsky, L., 2019, Repair of wind turbine blades: Review of methods and related computational mechanics problems, Renewable Energy, 140, 828-839

15. Mo J., LEE Y., 2012, CFD Investigation on the aerodynamic characteristics of a small-sized wind turbine of NREL PHASE VI operating with a stall-regulated method, Journal of Mechanical Science and Technology, 26, 81-92 
16. Powell S., 2011, 3M Wind Blade Protection Coating W4600, Industrial Marketing Presentation

17. Rempel L., 2012, Rotor blade leading edge erosion-real life experiences, Wind Systems Magazine, October

18. Sareen A., Sapre C.A., Selig M.S., 2012, Effects of leading edge protection tape on wind turbine blade performance, Wind Engineering, 36, 5, 525-534

19. Sareen A., Sapre C.A., Selig M.S., 2014, Effects of leading edge erosion on wind turbine blade performance, Wind Energy, 17, 1531-1542

20. Simms D., Schreck S., Hand M., Fingersh L.J., 2001, NREL unsteady aerodynamics experiment in the NASA-Ames wind tunnel: a comparison of predictions to measurements, National Renewable Energy Laboratory Golden, CO, Report No. NREL/TP-500-29494.

21. Sørensen G.S., 2009, Framework for risk-based planning of operation and maintenance for offshore wind turbines, Wind Energy, 12, 493-506

22. Wang Y., 2017, Research on the Effects of Leading Edge Erosion on Horizontal Axis Wind Turbine Performance, Lanzhou University, Lanzhou

23. Whang Y., Hu R., Zheng X., 2017, Aerodynamic analysis of an airfoil with leading edge pitting erosion, Journal of Solar Energy Engineering, 139, 6, 061002

24. Wang Y., Zheng X., Hu R., Wang P., 2016, Effects of leading edge defect on the aerodynamic and flow characteristics of an S809 airfoil, PLOS ONE, 11, 0163443

25. Zhang S., Dam-Johansen K., NørkJær S., Bernad P.L., Kill S., 2015, Erosion of wind turbine blade coatings - Design and analysis of jet-based laboratory equipment for performance evaluation, Progress in Organic Coatings, 78, 103-115 